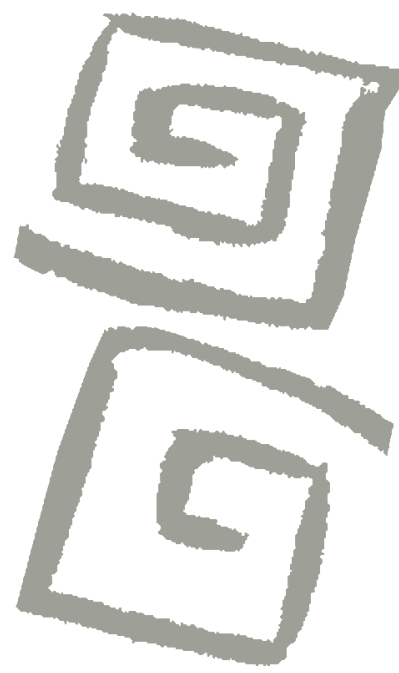

\title{
Trabajo, miserias y recompensas: Asistentes sociales, enfermeras y matronas en la construcción de la política sanitaria chilena a mediados del siglo $\mathrm{XX}$
}

\author{
Work, miseries and rewards: Social workers, nurses and \\ midwives in the construction of Chilean health policy in \\ the mid-twentieth century
}

Maricela González Moya', María Soledad Zárate Campos²

${ }^{1}$ Doctora en Historia. Académica Adjunta, Escuela de Trabajo Social, Pontificia Universidad Católica de Chile. Santiago, Chile. $\square$ iD

${ }^{2}$ Doctora en Historia. Académica, Departamento de Historia, Universidad Alberto Hurtado. Santiago, Chile. $\square$ (iD)
RESUMEN El artículo busca mostrar el aporte realizado por asistentes sociales, enfermeras y matronas a la exitosa política de salud pública de mediados del siglo XX en Chile, Ilevada a cabo por el Servicio Nacional de Salud en el marco de un modelo de desarrollo estatista y benefactor. Se han utilizado fuentes documentales de diverso tipo y testimonios de asistentes, enfermeras y matronas entrevistadas para la investigación. Las profesionales, encargadas fundamentalmente de tareas operativas y en contacto directo con la población usuaria del Servicio Nacional de Salud, fueron artífices de la instalación de una verdadera pedagogía sanitaria que cambió el patrón epidemiológico y trastocó la cultura de la población chilena, incidiendo en la valoración del autocuidado y la prevención. Como ejecutoras, sortearon las dificultades inherentes al trabajo burocratizado de los programas sociales pero, a la vez, recibieron gratificaciones diversas de tipo afectivo y altruista, que las hizo sentirse protagonistas de una construcción histórica.

PALABRAS CLAVES Asistente Social; Matrona; Enfermeras; Servicio Nacional de Salud; Chile.

\begin{abstract}
The article seeks to show the contribution made by social workers, nurses and midwives to the successful public health policy implemented in the mid-twentieth century in Chile by the National Health Service in the context of a statist and welfare development model. Documentary sources of different types and testimonies of social workers, nurses and midwives who were interviewed for this research were used. These professionals, mainly responsible for operational tasks and in close contact with the users of the National Health Service, were responsible for the establishment of a true health pedagogy that changed the epidemiological pattern and touched the culture of the Chilean population, influencing its estimation of self-care and prevention. As executors of the policy, they avoided the difficulties inherent to the bureaucratized work of social programs, gaining, at the same time, satisfaction at the affective and altruistic level, which made them feel like the protagonists of a historical process.
\end{abstract}

KEY WORDS Social Work; Midwifery; Nurses; National Health Service; Chile. 


\section{INTRODUCCIÓN}

A comienzos de la década de 1970, el Servicio Nacional de Salud (SNS) chileno se erguía como un organismo líder a nivel latinoamericano en términos de indicadores sanitarios, cobertura asistencial, formación de personal ejecutivo y directivo, producción de medicamentos y generación de una filosofía médica hermanada con el ideario social de la Organización Mundial de la Salud (OMS). En sus cortos veinte años de vida, había reducido drásticamente las tasas de mortalidad y morbilidad endémicas del país y había elevado todos los indicadores de calidad de vida de la población y, si bien seguía siendo superado por otros países de América y, por supuesto, por EEUU y Europa, el ritmo de mejoramiento era el más acelerado en mediciones como mortalidad materna e infantil, esperanza de vida, incidencia de enteritis y otras afecciones infecciosas y parasitarias ${ }^{(1)}$. La tuberculosis y las enfermedades de transmisión sexual, que durante la primera mitad del siglo XX habían sido causas principales de mortalidad en el país, se encontraban en rápida retirada y, a mediados de la década de 1960 , ya no se presentaban casos de viruela o tifus exantemático, mientras que disminuían drásticamente los enfermos de sarampión o poliomielitis, a causa de las masivas campañas nacionales de vacunación.

Estos logros sanitarios y sociales han sido atribuidos a múltiples factores, incluyendo el rol activo del Estado chileno, el espíritu avanzado de los médicos al implantar la doctrina de la medicina social que tuvo poderosos efectos redistributivos, la relativa estabilidad de la política sanitaria en un contexto de cambios sociopolíticos profundos y convulsionados, y las consecuencias que tuvo en la sanidad chilena el aumento generalizado de recursos públicos que permitieron expandir la cobertura de las prestaciones y multiplicar con abundancia las atenciones prestadas a la población.

Sin desconocer la importancia de todos estos elementos, el presente artículo busca mostrar el aporte realizado por la burocracia sanitaria chilena $y$, particularmente, por un grupo específico dentro de ella, conformado por las asistentes sociales, enfermeras y matronas que trabajaron en el SNS, ejerciendo el rol de implementadoras directas de la política de salud a través de tres mecanismos esenciales: 1) contacto directo "cara a cara" con las usuarias; 2) desarrollo de una pedagogía sanitaria que incidió en el cambio de patrones culturales de autocuidado de la salud; y 3 ) compromiso laboral con recompensas afectivas más que salariales, que desbordaron el rol que tenían prescrito y transcendieron a la propia política burocrática del SNS, generando efectos virtuosos para la salud del colectivo.

La bibliografía sobre el SNS es abundante y muy diversa, con predominio de las perspectivas institucionalistas, que ponen énfasis en la organización estructural del organismo, sus objetivos y aportes, y aquellas que ofrecen una visión panorámica sobre su inserción y contribución a la historia de la salud pública chilena ${ }^{(2,3,4,5)}$. Son enfoques generalistas, a menudo apologéticos y más o menos reiterativos. Escasean, sin embargo, los estudios sobre los profesionales que trabajaron en el SNS, pese a que los Cuadernos Médico Sociales y la Revista Médica de Chile publicaron algunos artículos académicos sobre ellos, además de las estadísticas de recursos humanos en fuentes de la época, incluyendo los anuarios sanitarios que se generaron en el propio SNS y en la Dirección General de Estadística ${ }^{(6,7,8,9,10)}$. El tema más recurrente fue la "escasez" de personal sanitario, las estimaciones cuantitativas sobre su distribución, y las estimaciones descriptivas respecto de sus roles en los distintos equipos de salud.

Los médicos fueron siempre objeto de estudio prioritario en la historiografía sobre profesiones sanitarias en Chile, tradición de la que han participado los propios médicos, con estudios de diversa calidad científica y aportando un cúmulo importante de escritos biográficos y autobiográficos ${ }^{(11)}$. Respecto de las matronas, enfermeras y asistentes sociales, ha surgido un interés por ellas en las últimas décadas, siendo las investigaciones de María Soledad Zárate ${ }^{(12,13,14,15)}$, Karin Rossemblatt $^{(16,17)}$, Maricela González ${ }^{(18,19,20,21)}$ y María Angélica Illanes ${ }^{(22,23)}$ las más relevantes. Un 
punto en común a todas ellas ha sido la reconstrucción del proceso de profesionalización de cada una de estas disciplinas y la perspectiva de género, que ha aportado una mirada crítica sobre su desempeño, los vínculos con otros profesionales y su apropiación particular de los procesos de cuidado y asistencia implicados en el quehacer médico.

En general, los estudios históricos sobre las profesiones en Chile no han sido abundantes y, tal como lo ha señalado Enrique de la Garza ${ }^{(24)}$, Latinoamérica ha llegado tarde a este campo de conocimiento, no obstante lo cual disponemos de algunos estudios sobre grupos profesionales específicos, principalmente médicos, ingenieros y profesores $^{(25,26,27,28,29,30)}$. En ese marco, el presente artículo pretende ser un aporte al conocimiento de varios procesos asociados: la construcción profesional dentro del campo de la salud pública, la edificación de roles y el estatus a partir de las asignaciones de género, la importancia de los implementadores de política en la cimentación del prestigio institucional del SNS y el modo en que todos estos elementos confluyeron en un momento específico de la historia chilena y latinoamericana.

La investigación se ha llevado a cabo usando fuentes de amplio espectro, lo que ha incluido material de archivo del SNS y publicaciones periódicas asociadas a la institución y a las profesionales de la medicina, la enfermería y la asistencia social. También incluyó la revisión de documentación institucional y gremial, legislación, decretos y oficios internos, monografías históricas, reportes internacionales y fuentes estadísticas.

Finalmente, se incorporaron algunos relatos biográficos de más de 50 entrevistas hechas a diferentes profesionales, cuyo valor testimonial ha sido de inconmensurable valía y trascendencia para la comprensión del proceso y le ha aportado la dimensión humana al estudio, imprescindible para valorar e indagar el tema. Todas las entrevistas fueron realizadas bajo consentimiento informado, aprobado por el Comité de Ética de la Universidad Alberto Hurtado. Tanto estas entrevistas como el trabajo documental llevado a cabo es parte de una investigación mayor titulada "Profesiones sanitarias femeninas en Chile 1950-1980. Prácticas, relaciones de género e identidades laborales", que cuenta con financiamiento del concurso Fondecyt Regular del gobierno de Chile.

\section{EL SERVICIO NACIONAL DE SALUD Y SU ROL SANITARIO EN CHILE Y LATINOAMÉRICA}

EI SNS fue creado por Ley de la República el 8 de agosto de $1952^{(31)}$, con un doble fin: por una parte, unificar las instituciones sanitarias existentes en el país, que habían ido creciendo y desarrollándose de modo inorgánico, generando una serie de trabas burocráticas debido a la falta de coordinación entre los organismos, la superposición de funciones y la carencia de una unidad de mando y dirección $^{(32)}$. Por otra parte, y estrechamente ligado con lo anterior, el SNS, tomando su inspiración de la doctrina de la medicina social y del ideario universalista de la OMS, no solo proporcionó una estructura organizada para la salud pública chilena, sino que la condujo con "unidad normativa y descentralización operativa", brindando una ruta clara con coherencia programática, presupuestaria y política ${ }^{(33)}$.

EI SNS fue la institución más representativa del Estado social chileno y el casi exclusivo proveedor de salud pública en el país. Brindó prestaciones curativas a más del $70 \%$ de la población y alcanzó cobertura total en acciones de saneamiento, protección y fomento de la salud. Absorbió cerca del $90 \%$ del presupuesto del sector salud y fue propietario de un $93,5 \%$ de las camas hospitalarias; triplicó su presupuesto real entre 1960 y 1972 y utilizó, ese último año, un 8,5\% del gasto fiscal total ${ }^{(1)}$.

EI SNS fue el organismo más grande e importante dentro de los servicios descentralizados del Estado chileno, con dependencia jerárquica del presidente de la República a través del Ministerio de Salud. A comienzos de la década de 1960 trabajaban en él un 
total de 44.068 funcionarios, los que constituían un $17,2 \%$ del total de la administración pública ${ }^{(34)}$. Desde su creación, y durante los veinte años siguientes, el SNS se fortaleció, incrementando su personal, su extensión territorial, sus programas y usuarios, siendo demandado de modo también incremental por sus beneficiarios legales y también por otros grupos sociales que se sentían atraídos por sus beneficios.

EI SNS puede ser considerado, en justicia, el organismo asistencial chileno por excelencia, inspirado en valores de atención integral y sostenido por un modelo de desarrollo estatista, benefactor, que se había asentado desde la década de 1930 en respuesta a las graves consecuencias que tuvo internamente la Gran Depresión ${ }^{(35)}$. Desde ese momento y hasta 1973, el Estado chileno fue internamente el principal actor político, social y económico. A nivel latinoamericano, y con la sola excepción de Cuba, Chile tuvo el sector público más significativo en cuanto a provisión de empleo, inversión y producción. La acción del Estado llegó a constituir un 30\% del PIB, las inversiones estatales sumaban más del $50 \%$ de todas las inversiones del país, las agencias estatales controlaban más de la mitad de los créditos, el empleo público cubría cerca de un $15 \%$ de la población económicamente activa ${ }^{(36,37,38)}$ y la Corporación de Fomento (CORFO), también estatal, poseía acciones en casi 80 de las principales empresas del país y era accionista mayoritaria en 39 de ellas ${ }^{(39)}$, a pesar de que el poder económico estaba igualmente concentrado en una pequeña élite ${ }^{(40)}$. El número de empresas públicas fue mayor que el de varios países europeos, llegando a 490 en 1972(37); al mismo tiempo, dominó el sistema sanitario y previsional, reguló la mayor parte de los procesos de negociación colectiva y vigiló salarios, precios, impuestos y tasas de importación ${ }^{(41)}$.

Afirmado en esta poderosa estructura estatal, el SNS cambió la fisonomía sanitaria chilena. Fue un continuador de algunos programas sanitarios y asistenciales previos, pero supo proyectar los buenos resultados, superar los déficits existentes y transformar el perfil epidemiológico de los chilenos, mejorando su calidad de vida y alcanzando indicadores sanitarios que se mantienen en el presente a la altura de países desarrollados ${ }^{(42)}$.

Durante la década de 1950, su principal logro fue mantener y mejorar algunos buenos resultados que había tenido la política pública chilena en los últimos decenios. El piso para conseguirlo fue el aumento de las atenciones en distintos niveles: consultas médicas, pediátricas y maternales; exámenes de laboratorio y radiológicos; kilos de leche entregados, vacunaciones, despacho de recetas y altas de hospitalización ${ }^{(43)}$. En los años siguientes, se produjo lo que Ernesto Medina, médico salubrista, describió como "una tendencia declinante sistemática de los riesgos de salud de los chilenos"(43). Entre 1925 y 1966 la mortalidad general disminuyó de 27,7 a 10,2 por 1.000 nacidos vivos, Ilegando a 6,9 en 1977. La mortalidad neonatal, que a mediados de la década de 1960 alcanzaba los 34,8 por 1.000 nacidos, bajó a 20,9, mientras que la mortalidad infantil general -uno de los indicadores más rebeldes en Chile- pasó de 258 en 1925 a 102,9 en 1964 , y a 47,5 en 1977 , siendo especialmente significativa la contención de la mortalidad infantil tardía, gracias a los programas de control del niño sano, vacunaciones y ataque a las enfermedades de mayor incidencia en la primera infancia ${ }^{(44,45,46,47)}$. La tasa de natalidad fue bajando acompasadamente con la marcha del programa de planificación familiar a mediados de la década de 1960, y en quince años descendió desde 36,2 por mil nacidos vivos a 22,6. El programa contribuyó también a paliar las altas tasas de mortalidad materna mediante la contención del aborto, pasando de 28,3 en 1964 a 10,3 a fines de la década de $1970^{(46)}$. Finalmente, la expectativa de vida, que en 1925 apenas alcanzaba a 36 años, en 1980 llegaba a un promedio de $69,3^{(48)}$.

Las razones de estos logros han sido discutidas abundantemente por una razón sencilla pero profunda: ¿cómo fue posible sostener tan buenos resultados en un clima político tan convulsionado como lo fue el de Chile durante el periodo de vida del SNS (1952-1980)?; ¿Cómo pudo el SNS sortear 
dichos avatares y mantener una línea de progreso continuo, habiendo pasado por cinco gobiernos muy diferentes, incluyendo el socialista de Salvador Allende y la dictadura militar de Pinochet?; ¿cómo se explican estos logros, siendo entonces Chile un país pobre, subdesarrollado, con una economía de escaso crecimiento y alta inflación?

El factor de la continuidad histórica fue clave. El SNS, habiendo sido un motor de cambios e innovador en doctrina y programas, fue al mismo tiempo un continuador de la política sanitaria y asistencial que se había iniciado en Chile a mediados de la década de 1920, con el dictado de las primeras leyes sociales y sanitarias, que fueron también vanguardia en América Latina. Sus programas más emblemáticos -medicina preventiva, atención madre y niño, control de enfermedades infecciosas- fueron impulsados $y$ mejorados por el SNS, pues se encontraban arraigados en la población y se habían visualizado sus efectos positivos. Por otro lado, la creación del SNS fue beneficiosa para el gremio médico y tanto ellos como los otros miembros del equipo sanitario vieron en la nueva institución un lugar para su desarrollo profesional y académico, razón por la cual el SNS se terminó convirtiendo en un espacio organizacional donde encontró cabida la filosofía de la medicina social y también las aspiraciones pragmáticas de los nuevos funcionarios.

Por otro lado, el incremento de la atención médica fue fundamental ${ }^{(42)}$ y la cobertura alcanzada se vio favorecida por otros factores ambientales relevantes. El más importante de ellos fue el nivel de alfabetización y educación de las madres, procesos que fueron impulsados desde mediados del siglo XIX y reforzados en la década de 1930, con el establecimiento de la educación primaria obligatoria $^{(49,50)}$. También fueron significativas las políticas de nutrición, pues contribuyeron a aumentar la resistencia a las enfermedades, favoreciendo el trabajo de las vacunas ${ }^{(47)}$; y el programa de planificación familiar, que permitió reducir las tasas de fecundidad $y$, consecuentemente, hizo que las familias tuvieran menos impedimento para trabajar $y$ que los recursos se aprovecharan de mejor manera $^{(45)}$.

Por último, ha sido considerado también el rol que tuvieron en este progreso sanitario los profesionales que crearon y gestionaron los diversos programas de salud del SNS. EI presente artículo pretende detenerse en este fenómeno, poniendo atención al estamento de implementadoras que tuvo a su cargo la operación de la política sanitaria chilena y el rol que jugaron en la institución.

\section{PROFESIONALES EN LA “LÍNEA DE FUEGO": DECISIONES, LIBERTAD Y EMOCIONES EN EL ACCIONAR SANITARIO}

El SNS fue el organismo de administración descentralizada más grande del Estado chileno y ocupó el segundo lugar -solo antecedido por el Ministerio de Educación- en número de empleados contratados. En su interior, tenía una composición tripartita, dividida de modo equitativo entre un estamento profesional, un estamento auxiliar de salud y un estamento administrativo.

Dentro de los profesionales, los médicos constituían cerca de un $45 \%$. Las matronas, asistentes sociales y enfermeras sumaban un $36 \%$ del grupo profesional: $18 \%$ las enfermeras, $11 \%$ las matronas y un $7 \%$ las asistentes sociales. Solo tuvieron jefaturas las enfermeras y las asistentes sociales, pues en la estructura del SNS se conformaron unidades de Enfermería y Servicio Social como parte de los Servicios Técnicos Generales, dependientes de la Subdirección Normativa del Servicio $^{(51)}$. Las matronas tenían un rol más acotado y operativo aún y, por ende, no estuvieron adscritas a un departamento, sino a programas de acción y, en particular, al programa de salud materno-infantil.

EI SNS favoreció la formación y el empleo de estas tres profesionales, pues mantuvo escuelas superiores de servicio social, enfermería y obstetricia, además de las que existían a nivel universitario. Fue también un empleador relevante para las asistentes 
sociales (cerca de un 30\% de las profesionales activas trabajaban en el Servicio) ${ }^{(52)}$ y casi exclusivo para enfermeras $(91,4 \%)^{(52)}$ y matronas $(92 \%)$, aunque estas últimas podían complementar su desempeño con la atención de partos a domicilio de forma particular ${ }^{(53)}$.

La formación de matronas y enfermeras las habilitaba para actuar directamente en la reparación y rehabilitación de la salud de los pacientes y en la atención obstétrica. Realizaban tareas de curación, higiene, suministro de medicamentos, aplicación de tratamientos, atención del recién nacido y realizaban todos los procedimientos terapéuticos que eran indicados por los médicos, siendo ellas las encargadas de "dar cumplimiento a dichas indicaciones"(54). Eventualmente podían incluso verse limitadas en su actuar, como fue el caso de la matrona Ema Osorio, que testimoniaba haber recibido una formación avanzada, bajo reglas de asepsia y registro modernas pero que, al ingresar a trabajar al Hospital de Punta Arenas, tuvo "problemas" porque "no nos permitía el médico hacer la ficha clínica" (entrevista Ema Osorio, 2011) y, por ende, el trabajo se reducía a cumplir órdenes estrictas de mera ejecución práctica.

Las asistentes sociales, por su parte, cuya historia había estado tradicionalmente ligada a la atención de necesidades asistenciales y también habían desarrollado un temprano vínculo con el ámbito sanitario ${ }^{(21)}$, recibían una formación que implicaba la realización de residencias prácticas en diversas unidades del SNS y, en el año 1956, a través del dictado de las normas que señalaban las funciones de la Sección de Servicio Social, se especificaron un conjunto de deberes en las zonas y centros de salud, hospitales, consultorios y hogares de menores ${ }^{(55)}$. Trabajaban mayoritariamente en hospitales del Servicio (cerca de un $80 \%$ de ellas) ${ }^{(7,9)}$, donde cumplían una rutina asociada al ingreso, tratamiento y egreso de los pacientes, realizando también visitas y trámites generales para la gestión de beneficios, subsidios y pensiones ${ }^{(56)}$. Por otro lado, fueron agentes comunitarias por excelencia, pues habían incorporado desde la década de 1940 el método de intervención comunitaria como parte de su instrucción, insertándose en los barrios populares para el apoyo de organizaciones vecinales, creación de grupos coordinados para la gestión de recursos, formación de líderes, entre otras tareas ${ }^{(57)}$.

Es evidente que las enfermeras, matronas y asistentes sociales no fueron las únicas profesionales que trataron directamente con el público beneficiario del SNS, pero su particularidad estuvo en lo que Lipsky ha denominado la realización de una intervención "en la línea de fuego"(58), esto es, constituirse en ese grupo de funcionarios ejecutores de la política pública que interactúan directamente y la mayor parte del tiempo con el público, sirviendo de receptores de todo un conjunto de interacciones producidas en los servicios y gozando, a la vez, de un alto grado de discrecionalidad en la aplicación de los programas.

Casos cotidianos del operar del SNS nos sirven de ilustración: los médicos atendían pacientes, decidían los tratamientos que se suministraban, establecían las normas para resolver problemas de salud con base en el conocimiento científico disponible y en su propia experiencia terapéutica. Pero quienes ejecutaban dichas disposiciones eran enfermeras, matronas y asistentes sociales, y en la distancia que mediaba entre la orden médica y su aplicación se encontraba todo un universo de opciones específicas que daban "vida" a la prescripción médica. Por ejemplo, eran estas profesionales las que "traducían" las indicaciones a un lenguaje que fuera entendible y cercano para los pacientes, usualmente personas con bajo nivel de instrucción y a menudo atemorizadas por la enfermedad (entrevista Mirta Rojas, 2013). La asistente social Teresa Hernández lo expresaba con claridad: "yo iba detrás del médico y anotaba todo lo que él decía en su ronda por las salas [...] y luego les mostraba a las familias cómo debían darse los remedios, les contaba las horas y les mostraba la importancia de seguir el tratamiento" (entrevista Teresa Hernández, 2012). Eleonor Codoh, que acudía con un grupo de niños al Consultorio de Chiguayante (Concepción), cumplía el mismo rol, escuchando y registrando lo que debían realizar, y luego se reunía con cada una de 
sus madres y les explicaba, "en palabras sencillas", lo que el médico había dicho, "para que los campesinos pudiesen acatar las órdenes y los niños se mejoraran"(59).

Otra dimensión era la resolución de problemas. La enfermera Isabel Ringeling señalaba que, en los turnos de noche del Hospital Roberto del Río, el grupo que ella comandaba de auxiliares y matrona acordaban disposiciones que escapaban por completo a su ámbito de competencia, y que habían decidido, aun en contra de la tendencia de la época, dejar que las madres entraran a cuidar a sus hijos porque se habían dado cuenta de que eso mejoraba el proceso de recuperación (entrevista Isabel Ringeling, 2017). Cecilia Moya, enfermera en la sección de quemados del mismo hospital, relataba haber Ilorado con los niños cuando a estos les dolían las curaciones y conmovida, había logrado que los médicos "les pusieran un calmante antes de que los fueran a curar, porque los niños sabían que era día de curación y empezaban a sufrir en la mañana"; los médicos "me echaban", señalaba, pero ella persistía: "no me voy, les decía, me quedo aquí al lado del niño, lloraba yo por el niño, pero me hacían caso, era tan escandalosa [...] además lograba que otras colegas se entusiasmaran conmigo y también alegaban" (entrevista Cecilia Moya, 2017).

La escasez de médicos daba también un margen de actuación más amplio, pero también más arriesgado, aunque desafiante. La matrona Margarita Bravo dijo haber tenido claridad respecto de cuál era el límite de su trabajo, pero que era frecuente sobrepasarlo por falta de implementos, medicamentos $u$ horas médicas: "...cualquier patología que hubiera en el parto teníamos que darle cuenta a ellos" (entrevista Margarita Bravo, 2011), pero si él no estaba y la urgencia era inminente, la matrona debía actuar con rapidez, aun si se trataba de un procedimiento estrictamente quirúrgico: retención de placenta, transfusión de sangre, administración de anestesia, entre otras (entrevista Graciela Bravo, 2011). Ellas y otras matronas daban cuenta también de los contrastes que se producían entre los diversos servicios e, incluso, entre los diferentes médicos que los dirigían. Ema Osorio dice haber tenido funciones y responsabilidades muy distintas mientras trabajó en el Hospital de Punta Arenas, pues habiendo llegado a un ambiente bastante tradicional, con "matronas viejas" y un médico que "no quería cambiar nada", había pasado posteriormente a ser dirigida por un nuevo facultativo que amplió sus tareas y le dio más formalidad al trabajo (entrevista Ema Osorio, 2011).

Ser implementadoras les aseguraba un contacto directo con las personas usuarias del SNS -mayoritariamente mujeres- y las ponía en contacto con su vida cotidiana, con sus pesares y alegrías y también les permitía contribuir estratégicamente a la construcción del vínculo con sus hijos, en la medida en que los índices de mortalidad infantil descendían ${ }^{(60)}$. El Servicio atendía a personas pobres y las carencias de salud se confundían con las malas condiciones de la vivienda, la alimentación o la escolaridad. La asistente social Victoria Romeo menció haber sentido muy profundamente lo que era el "olor de la pobreza", una mezcla de humedad, encierro, tierra en las calles y barro en invierno, y dijo haber enseñado a los médicos jóvenes "cómo se hacía medicina en la casa de los pobres", recorriendo las poblaciones hasta tarde en la noche o los fines de semana, entrando en las viviendas de los moribundos y tomando en sus brazos a los niños desnutridos (entrevista Victoria Romeo, 2012).

El contacto directo con las usuarias convertía a las profesionales en lo que Mark Graham ha denominado "burocracia emocional"(61). Atender la salud era también escuchar, e incluso sostener, un flujo de emociones que desbordaban los márgenes definidos para la actuación técnica. La asistente social Eliana Iglesias testimonia haber hecho la solicitud para tener una oficina "donde poder ubicarme y poder tener cierta privacidad", porque las personas necesitaban "respeto" y tenían una especial necesidad de ser escuchadas. Las usuarias, decía Eliana, Ilegaban "con algún problema, con una emoción adentro" y había que saber "cómo orientarlas" (entrevista Eliana Iglesias, 2016). Y Felicidad Heredia, otra asistente, señalaba que había aprendido "cómo ir orientando a una persona que 
venía muy angustiada, dejarla por lo menos que se desahogara" (entrevista Felicidad Heredia, 2012).

Efectivamente, el factor "orientación" era clave. Una parte importante del público eran personas que podían provenir de sectores muy marginales o del campo. Aunque esos fuesen casos extremos, frente a ellos la intervención se desplazaba más allá de los "límites profesionales"(62) y en los relatos de las entrevistadas abundan los ejemplos: acompañar el funeral de un niño que fue abandonado, conseguir vestuario o alimentos para situaciones de extrema precariedad, gestionar alojamiento para una familia que venía de fuera de Santiago o tramitar medicamentos que el público no pudiese adquirir. Estas acciones, denominadas "expansión de fronteras" por Meredith Honig ${ }^{(62)}$, son propias también de los implementadores y producen, efectivamente, resultados inesperados de la política o aceleran sus logros porque se ajustan a las necesidades de los usuarios de manera efectiva y potente, generando eficiencia en los resultados y legitimando programas sociales que aparecen humanos, cercanos, sensibles frente a los usuarios.

Las visitas domiciliarias fueron esenciales para obtener efectos sanitarios en tan poco tiempo y fueron parte del cotidiano de trabajo de las matronas y las asistentes sociales. Como se ha descrito en algunos relatos, las asistentes realizaban verdaderas rondas de vigilancia en los barrios populares, rastreando la existencia de embarazadas que no hubiesen aún asistido al control prenatal (entrevista María Oxman, 2014). A otras se las visitaba hasta convencerlas y mientras la matrona instruía sobre los beneficios de la atención institucional e inspeccionaba el estado de salud de la mujer, la asistente se ocupaba de la situación socioeconómica general, indagando sobre los problemas familiares, habitacionales y económicos ${ }^{(63)}$.

Las matronas acompañaban también las rondas domiciliarias de los médicos generales de zona, enviados a residir en áreas rurales por algunos años como parte de su carrera profesional. La falta de recursos, la lejanía de los sectores rurales, la resistencia que ponían las comunidades ante la llegada de los servicios de salud, hacían de este trabajo una verdadera cruzada apostólica y transformó a las matronas en funcionarias polifuncionales que atendían las múltiples necesidades comunitarias, sorteando obstáculos culturales y enormes vicisitudes prácticas. Ellas mismas lo calificaban como una especie de cruzada agotadora y ponían de manifiesto que se encontraban cansadas, con escaso tiempo para participar de actividades de esparcimiento y a menudo aisladas de otros profesionales, sorteando "todas las deficiencias que existían en ese tiempo" para trasladarse a pequeñas aldeas rurales, falta de transporte adecuado y extensas jornadas (entrevista Ema Gómez, 2011). Sin embargo, el SNS puso mucho énfasis en organizar la distribución de matronas a lo largo del territorio chileno, porque su trabajo era considerado fundamental en el Programa Materno Infanti $i^{(64,65,66)}$ y en varias localidades, quedando ellas a cargo en forma exclusiva de los centros de salud, donde debieron combinar sus competencias técnicas con habilidades de administración y mando ${ }^{(53)}$.

Matronas y asistentes enlazaban a la familia con la red de recursos institucionales y legales existentes, insertándolas en un dispositivo sociopolítico que era, a la vez, una ayuda para mejorar su vivir y un mecanismo de sometimiento a la estructura familiar, social y cultural dominante ${ }^{(16,17)}$. Un ejemplo nos sirve para ilustrar el proceso: según el relato de María Ester Pérez, estudiante en práctica de Servicio Social en el Consultorio Rosita O'Higgins de la ciudad de Concepción, se realizaron en el mes de febrero de 1975 una serie de actividades semanales de formación y motivación con contenidos sanitarios; participaron enfermeras, asistente social, ingeniero sanitario y nutricionista y se trataron temas referidos a la prevención de diarreas estivales y, adicionalmente, se dictó una charla sobre los "Beneficios del Servicio Nacional de Salud". Además de la entrega de información, se entretuvo a las personas a través de la exhibición de películas y la demostración práctica para la "preparación de leche en polvo", "preparación higiénica de mamaderas" y "control del niño sano"(67). 
De esta manera, la intervención de este ejército de ejecutoras terminaba por regular la vida de las familias obreras. Sobre todo, mediante la creación y funcionamiento de clubes de madres y embarazadas (entrevista Elena Valladares, 2013), se instalaba una pedagogía sanitaria que instruía a las mujeres sobre la crianza, los valores de la maternidad, la organización de la economía doméstica e incluso sobre derechos sociales y previsionales ${ }^{(68)}$. En el programa de planificación familiar, por ejemplo, el rol de las profesionales era esencialmente "educativo" y se debían mostrar las bondades de una proyección racional de la familia ${ }^{(69,70)}$ y tanto en los clubes y centros de madres, como en las visitas al domicilio, se entregaban todo tipo de indicaciones para normar la cotidianeidad. Mafalda Ortiz señalaba haber creado clubes en la zona urbana de Chillán no solo para enseñar las bondades del control de salud y la higiene de la vivienda, sino también para inducir a las mujeres a cobrar la asignación familiar obrera y mostrarles cómo los niños debían jugar de modo sano y ser castigados adecuadamente ${ }^{(71)}$. Lida Valladares, otra asistente, contaba con admiración, haber visto a una colega visitar un hogar pobre $y$, en ausencia de la madre, haber limpiado la casa, aseado a los niños y preparado el almuerzo, luego de lo cual le había dicho a la mujer, cuando regresó: "así debe estar la casa para obtener los beneficios que está solicitando" (entrevista Lida Valladares, 2012). Al mismo tiempo, Isabella Kaplan, en el Centro de Salud de Viña del Mar, describía su trabajo asociado a la realización de rondas grupales por diversos hogares, donde las mujeres presenciaban lecciones de economía doméstica: se les enseñaba a preparar adecuadamente los alimentos y se nombraba una comisión de "atención de niños", formada por un grupo de socias que eran "supervisadas constantemente por la líder, como un medio de impartir nociones de cuidado al menor" ${ }^{\prime \prime 63)}$.

\section{Pesares y carencias en la ejecución de la política sanitaria: Las profesionales en la red burocrática}

A fines de la década de 1960, cuando el SNS estaba ya plenamente consolidado, las profesionales en estudio constituían una pequeñísima minoría letrada en el contexto global del país. La población total era de 8.884.768 personas y las mujeres constituían un 51,1\% de ella ${ }^{(6)}$. Un $10,8 \%$ de las mujeres era analfabeta, un $61,9 \%$ poseía algún tipo de instrucción básica, un 20\% tenía algún tipo de instrucción secundaria, un 4,7\% instrucción técnica o normalista y solo un $2,75 \%$ de la población femenina de 15 años o más había ingresado (no necesariamente había finalizado) a la formación universitaria ${ }^{(6)}$. Si analizamos el mercado del trabajo, la participación laboral femenina alcanzaba el $23,1 \%{ }^{(6)}$ y un $11 \%$ aproximadamente correspondía a empleos profesionales ${ }^{(72)}$, estimándose una cantidad aproximada de 73.690 mujeres profesionales trabajando a comienzos de la década de 1970. Asistentes sociales, enfermeras y matronas representaban cerca de un $13 \%$ de ellas y, a su vez, 4.140 trabajaban en el SNS ${ }^{(53)}$.

Sin embargo, ni su estatus profesional ni el hecho de que constituyeran una verdadera élite ilustrada dentro del país en general, y al interior de la fuerza laboral femenina en lo particular, lograron hacer que se sintieran plenamente satisfechas ni reconocidas al interior del SNS. Como se ha dicho anteriormente, estaba definido formalmente que su responsabilidad era exclusivamente operativa. Ninguna de ellas rehuía al trabajo aplicado, pero recelaban del escaso reconocimiento que recibían y a menudo se sentían obligadas a realizar tareas poco específicas que no estaban a la altura de sus conocimientos técnicos ${ }^{(52)} \mathrm{O}$, como en el caso de enfermeras y matronas, que fácilmente se confundían con actividades domésticas ${ }^{(73)}$. Estas actividades eran atribuidas por algunos médicos a su condición femenina, pues requerían "además de conocimientos y de experiencia, una constitución particular que es más propia del espíritu de la mujer"(74). Revisar la disponibilidad de camas hospitalarias, organizar turnos, revisar y 
solicitar materiales para las salas, mantener inventarios, velar por la estética y la higiene de los servicios ${ }^{(73)}$ molestaba a muchas profesionales y, como lo señalaba Orieta Álvarez, asistente social que a mediados de la década de 1960 había organizado un estudio en la Escuela de Salubridad de la Universidad de Chile: "a 40 años de la creación del Servicio Social en Chile, pese a todos los esfuerzos realizados tendientes a una preocupación profesional, los asistentes sociales del SNS aún no son utilizados plenamente, y se desconoce su valer profesional"(52).

Como parte de su reclamo por reconocimiento, sostenían que una parte importante del trabajo administrativo no se correspondía con los conocimientos que habían adquirido en su formación -muchas de ellas eran ex alumnas del propio SNS-, ni con la disciplina o el rigor en los que habían sido instruidas. Como se evidenciaba en la investigación llevada a cabo en 1970 sobre los roles en el equipo de enfermería, las enfermeras mostraban gran "insatisfacción [...] con el rol que la organización de salud le ha impuesto, que al parecer se aleja bastante del rol para el cual fue entrenada", básicamente por el "desplazamiento hacia las labores administrativas y su consiguiente desagrado por estas"(72). No era para ellas ni para las matronas o las asistentes, una falta de pericia o interés por la rutina burocrática, sino más bien un enojo con lo que ese hecho significaba para su identidad profesional, la que se desperfilaba y creaba "confusión sobre las verdaderas funciones de este profesional, ya sea en el equipo de salud, frente al público, como también en alguna medida al desconocimiento del rol"(75).

La dependencia absoluta respecto del poder médico era otro factor de resentimiento. Aunque la burocracia chilena se regía por un estatuto administrativo que regulaba el empleo público y, en teoría, facilitaba la carrera funcionaria y el ascenso por mérito, en algunos organismos se producían verdaderos feudos profesionales pues para cualquier función superior o cargo directivo se necesitaban conocimientos especializados o se definía la disciplina que debía poseer: los ingenieros y arquitectos en el Ministerio de Obras Públicas, los profesores en el de Educación, los agrónomos en el de Agricultura, eran ejemplos notables de esta suerte de monopolio ${ }^{(41)}$. En el SNS, los médicos no solo ocupaban los más altos cargos (la Dirección General, las Subdirecciones Generales y Zonales y las Direcciones y Subdirecciones de todos los servicios y centros de salud), sino que poseían un estatuto particular con horarios y remuneraciones sujetos a condiciones muy superiores ${ }^{(76)}$. Sin embargo, las enfermeras reclamaban que la presencia de los directores médicos en los consultorios era mínima, con muy pocas horas de trabajo y con desconocimiento de la vida diaria del centro de salud (entrevista Iris Espinoza, 2017); mientras, las asistentes los describían como "superiores, arrogantes" (entrevista Victoria Romeo, 2012) ajenos a las necesidades de los pacientes y lejanos a los equipos de salud. Los médicos, según palabras del antropólogo Claudio Sepúlveda, constituían en el SNS una verdadera "subcultura particular" porque, aunque pertenecían al mismo estamento que los otros miembros del equipo, se regían por reglamentos especiales, poseían un estatus diferente, se comportaban de acuerdo a normas propias y se organizaban de modo especial (77). Eran, más que "jefes", verdaderos "señores", con sus compañeros de trabajo "a su servicio"(77).

Este "señorío" podía ser aceptado y encontrarse naturalizado dentro de los profesionales de colaboración médica. A fin de cuentas, había sido siempre de esa manera y se había perpetuado por la cultura organizacional y la tradición sanitaria. Sin embargo, eran sus consecuencias las que generaban resentimiento, por ejemplo, carecer de autoridad sobre los otros miembros del equipo y tener que esperar la orden del médico para entregar instrucciones o dictaminar sanciones. Enfermeras y asistentes sociales conformaban subdepartamentos con una jefatura de su mismo oficio, pero esas unidades no poseían ninguna responsabilidad que les otorgara poder: no tenían potestad presupuestaria, no tenían discrecionalidad en la toma de decisiones ni entregaban órdenes directas al personal que estaba a su cargo. En todas 
aquellas funciones donde les correspondía "dirigir", "organizar", "desarrollar", se hacía énfasis en hacerlo "de acuerdo con las normas establecidas"(73) y sometiendo todo tipo de decisión al cargo administrativo superior.

No obstante, debían ser jefas y tomar decisiones estratégicas cuando enfrentaban el trabajo cotidiano de sanidad y asistencia. Varios relatos de enfermeras señalan haber sido ellas quienes quedaban a cargo de los servicios de salud cuando los médicos se ausentaban y dado que estos cumplían jornadas muy estrechas de trabajo, era de su competencia, aunque no existiera reconocimiento formal de aquello, dirigir las acciones de salud y administrar el funcionamiento general de los consultorios y postas (entrevista Iris Espinoza, 2017). Los médicos estaban en condiciones de dirigir el trabajo general de la salud pública chilena, pero en lugares periféricos, en turnos vespertinos o en ausencia de la dirección general, el trabajo quedaba dirigido, organizado y supervisado por las enfermeras más competentes (entrevista Isabel Ringeling, 2017). En el mundo rural, en las postas y pequeñas estaciones de atención, no era raro encontrar matronas dirigiendo el trabajo ${ }^{(78,79)}$ y tanto ellas como otras enfermeras improvisaban nociones de administración sanitaria o participaban de los cursos que dictaba la Escuela de Salubridad para adquirir competencias de organización y gestión del quehacer sanitario.

Otro aspecto que tensionaba su trabajo era la escasez de personal sanitario, problema que venía siendo presentado desde la década de 1950 y que se fue agudizando de modo progresivo en los años siguientes. Para el común de los analistas y para una parte del mundo político, el aumento de la burocracia estatal que se había producido en los últimos decenios era algo escandaloso e intolerable y generaba vicios administrativos que dañaban el presupuesto y la imagen del país. Internamente, en cambio, las autoridades del SNS planteaban insistentemente que los fines de la política sanitaria no podían cumplirse si no se llenaban los puestos vacantes $y$, aun así, el déficit de profesionales seguiría siendo agudo y casi insalvable.
La escasez de médicos era un problema grave, pero el de enfermeras era verdaderamente crítico. En las escuelas profesionales se graduaban cerca de 130 nuevas enfermeras al año, lo que daba un total estimado de 2.428 enfermeras activas al año $1968^{(53)}$ y un déficit de 6.000, de acuerdo a las estimaciones hechas por el investigador Julio Morales, del Centro Latinoamericano y Caribeño de Demografía $(C E L A D E)^{(80)}$. Esta escasez incidía muy negativamente en la mala distribución de enfermeras en el país e incluso producía importantes carencias en lugares donde se las requería de manera urgente, como eran los servicios pediátricos y las visitas domiciliarias $^{(53)}$. Por otra parte, la creación de nuevas escuelas no solucionaba del todo el problema, pues los requisitos de ingreso eran exigentes, la motivación para estudiar la carrera escasa y la deserción estudiantil alta, debido a la precariedad de docentes preparadas y la falta de campos clínicos adecuados ${ }^{(53)}$.

En cuanto a las matronas, eran también un recurso escaso, pero se encontraban mejor distribuidas a lo largo del territorio ${ }^{(53)}$. Sin embargo, hacia 1970 se calculaba un déficit de $53 \%$ y se ponían en evidencia otras carestías: por tratarse de un oficio esencialmente clínico, a pesar de su antigüedad, la profesión en Chile ${ }^{(12)}$ había logrado muy poco desarrollo disciplinario y la mayor parte de las matronas tenía una posición subordinada, sin atribuciones directivas y el desempeño de su trabajo, muy demandante y sujeto a permanentes imprevistos, impedía su perfeccionamiento y mejoramiento ${ }^{(53)}$. Como señalaba el gremio, las matronas, y en particular las que se desempeñaban en zonas rurales, trabajaban excesivamente, tenían poco tiempo para perfeccionarse y a menudo debían lidiar con la escasez de insumos y materiales $y$, aunque suene redundante, con la falta de tiempo para hacer trabajo comunitario y educar a las usuarias $^{(53)}$.

Las asistentes sociales, por su parte, eran también un recurso escaso para el SNS y su déficit se estimaba en un $43 \%$, existiendo algunos cargos sin llenarse ${ }^{(52)}$. Las dificultades que enfrentaban eran similares a las de enfermeras y matronas y se podrían resumir, 
para todas ellas, en las siguientes: sobrecarga de trabajo, debido a la creciente demanda de atención sanitaria que tuvo el SNS a medida que pasó el tiempo; falta de reconocimiento y valoración de su trabajo, lo que se traducía en bajos salarios, carencia de una carrera atractiva e insuficiencia de incentivos para el perfeccionamiento; ambigüedad en sus roles y funciones por la realización de tareas múltiples, que venían a tapar los déficits de recursos humanos que tenía el SNS, pero que terminaban por subvalorar los conocimientos especializados que tenían, y exceso de burocratización del desempeño, con una gran cantidad de tiempo dedicado a tareas administrativas menores $y$, por contrapartida, sensación permanente de falta de tiempo y recursos para realizar tareas que aportaran efectivamente al desarrollo sanitario global del país.

\section{CONCLUSIONES}

La realidad profesional de las asistentes sociales, matronas y enfermeras en el SNS fue la propia de muchos implementadores de política, tal como muestra la bibliografía sobre el tema. Sobrecarga de trabajo, falta de reconocimiento, ambigüedad de funciones y ausencia de atribuciones directas de autoridad fueron sus reclamos permanentes y tensionaban el actuar de la institución en múltiples frentes.

Sin embargo, su rol en la construcción y ejecución de dicha política y en la consecución de sus logros históricos fue fundamental, y sus recompensas laborales no estuvieron asociadas al estatus o a las remuneraciones, sino al contacto directo que mantuvieron con las usuarias del Servicio. La intervención sanitaria y social producía una interacción educativa y afectuosa, fuertemente cargada de mesianismo e impregnada de valores altruistas y edificantes.

Las profesionales instalaron una pedagogía sanitaria entre mujeres y, a través de ella, se sembró en las familias chilenas una cultura del autocuidado cuyos frutos crecen hasta la actualidad. Probablemente sin quererlo del todo, empoderaron a las clases populares, haciéndolas conscientes de sus derechos frente a la institucionalidad pública. A la vez, promovieron la asociatividad, reunieron a las madres con un fin instructivo y sostuvieron la relevancia de la participación en la resolución de sus problemas. Promovieron el trabajo comunitario en salud $y$, trasladándose por una tupida red en el territorio nacional, Ilevaron salud hasta remotos lugares. Como resumía la matrona Ema Gómez:

\footnotetext{
La cultura generó un cambio de paradigma fácilmente [...] bastante más rápido de lo que nosotros o de lo que los directivos se propusieron. Nuestras mujeres fueron muy claritas y entendieron muy fácil, porque nosotros éramos los agentes educativos [...] entendieron muy bien que había que controlarse el embarazo, que había que ser responsables, que había que tener los hijos que se podía tener. (entrevista Ema Gómez, 2011)
}

Sin embargo, más que estar atentas a sus aportes, las profesionales se vieron sobre todo gratificadas afectivamente y recuerdan hoy lo que significó, en su vida laboral, haberse comprometido férreamente y haber trabajado con fuerza y firmeza por la salud de las personas. Como afirmaba la enfermera Cecilia Moya, a modo de colofón: "hicimos un trabajo heroico" (entrevista Cecilia Moya, 2017). 


\section{AGRADECIMIENTOS}

El presente artículo recoge algunos hallazgos parciales obtenidos de la investigación "Profesiones sanitarias femeninas en Chile 1950-1980. Prácticas, relaciones de género e identidades laborales". El estudio ha sido posible gracias al financiamiento recibido por parte de la Comisión Nacional de Investigación y Tecnología (CONICYT) del gobierno de Chile, Código de Proyecto $N^{\circ} 1161204$, Concurso Fondecyt Regular, otorgado el año 2016. Las autoras agradecen también a los encargados de los diferentes repositorios que han facilitado acceso a fuentes documentales y a todas las enfermeras, asistentes sociales y matronas entrevistadas para esta investigación, cuyo valioso testimonio ha enriquecido la visión del periodo en estudio.

\section{REFERENCIAS BIBLIOGRÁFICAS}

1. Organización Panamericana de la Salud. Las condiciones de salud de las Américas 1973-1976. Washington: OPS; 1978.

2. Horwitz N. Salud y Estado en Chile: Organización social de la salud pública, periodo del Servicio Nacional de Salud. Santiago: OPS; 1995.

3. Illanes MA. En el nombre del pueblo, del Estado y de la ciencia, (...). Historia social de la salud pública, Chile 1880-1973. Santiago: Colectivo de Atención Primaria; 1993.

4. Monasterio H, Urriola R, Massardo J, Molina CA. Historia de la protección social de la salud en Chile. Santiago: FONASA; 2009.

5. Molina C. Institucionalidad sanitaria chilena, 1889-1989. Santiago: LOM; 2010.

6. Instituto Nacional de Estadísticas. Censo 1970. Santiago: INE; 1970.

7. Dirección General de Estadísticas. Estadística Chilena, Asistencia Social, 1952-1960. Santiago: Dirección General de Estadísticas; 1952-1960.

8. Instituto Nacional de Estadísticas. Anuario de Atenciones y Recursos. Santiago: INE; 1970-1975.

9. Servicio Nacional de Salud Sub-Departamento de Estadística. Anuario de Atenciones y Causas de Muerte. Santiago: SNS; 1971-1981.

10. Servicio Nacional de Salud Dirección de Estadísticas y Censos. Anuario de Atenciones y Recursos. Santiago: SNS; 1965-1980.
11. Zárate Campos MS, del Campo AP. Curar, prevenir y asistir: Medicina y salud en la historia chilena. Nuevo Mundo Mundos Nuevos. 2014. doi: 10.4000/nuevomundo.66805.

12. Zárate MS. Dar a luz en Chile, siglo XIX: De la "ciencia hembra" a la ciencia obstétrica. Santiago: Ediciones Universidad Alberto Hurtado; 2007.

13. Zárate MS. Embarazo y amamantamiento: cuerpo y reproducción en Chile. En: Góngora A, Sagredo R, (dirs.). Fragmentos para una historia del cuerpo en Chile. Santiago: Taurus; 2010.

14. Zárate MS. Al cuidado femenino. Mujeres y profesiones sanitarias, 1889-1950. En: Stuven AM, Fermandois J, (eds.). Historia de las mujeres en Chile. Tomo II. Santiago: Taurus; 2013.

15. Zárate MS, González M. Matronas y parto hospitalario en Chile, 1950-1970. En: Sánchez G, Laako H, (eds.). Parterías de Latinoamérica: Diferentes territorios, mismas batallas. México DF: El Colegio Frontera Sur; 2018.

16. Rosemblatt K. Gendered compromises: political cultures, socialist politics and the state in Chile, 1920-1950. Chapel Hill: The University of North Carolina Press; 2000.

17. Rosemblatt K. Por un hogar bien constituido: El Estado y su política familiar en los Frentes Populares. En: Godoy L, (ed.). Disciplina y desacato: construcción de identidad en Chile, siglos XIX y XX. Santiago: SUR, CEDEM; 1995.

18. González Moya M. Inside Home: La visita domiciliaria de trabajadores sociales como estrategia de vinculación entre orden institucional y familia, Chile, 1925-1940. AMMENTU. 2014;1(4):241-243.

19. González M, (ed.). Historias del Trabajo Social en Chile, 1925-2008: Contribución para nuevos relatos. Santiago: Ediciones Técnicas de Educación Superior; 2010.

20. González M. Vino nuevo en odres viejos: Servicio Social de mediados de siglo XX en Chile: La construcción de la consolidación profesional, 19501973. En: Lizana R, (comp.). Trabajo social e investigación. Buenos Aires: Espacio Editorial; 2014.

21. González M. Asistentes sociales y salud pública en Chile: identidad profesional y lucha gremial, 1925-1973. Revista Dynamis. 2017;37(2):345-365.

22. Illanes MA. Ella en Lota-Coronel: poder y domesticación: El primer servicio social industrial en América Latina. Revista Mapocho. 2001;(49):141148. 
23. Illanes MA. Cuerpo y sangre de la política: La construcción histórica de las visitadoras sociales en Chile, 1887- 1940. Santiago: LOM; 2007.

24. Garza E. Tratado latinoamericano de sociología del trabajo. México: Fondo de Cultura Económica; 2000.

25. González Leandri R. Campos e imaginarios profesionales en América Latina: Renovación y estudios de caso. Anuario IEHS. 2006;(21):333-344.

26. Frederic S, Graciano O, Soprano G. El Estado argentino y las profesiones liberales académicas y armadas. Rosario: Prohistoria; 2010.

27. Villaseñor García G, (comp.). Las profesiones en México. México DF: UAM-Xochimilco; 1992.

28. Arce Gurza F, Bazant M, Staples A, Vázquez JZ. Historia de las profesiones en México. México: El Colegio de México; 1982.

29. Pérez Perdomo R. Medio siglo de historia judicial en Venezuela. Cuadernos Unimetanos. 2007(11):3-24.

30. Plotkin M. Notas para un análisis comparativo de la constitución del campo de los economistas en Argentina y Brasil. Anuario IEHS. 2006; (21):467-494.

31. Chile, Ministerio de Salubridad, Previsión y Asistencia Social. Ley 10383 de 8 agosto 1952, que crea el Servicio de Seguro Social y el Servicio Nacional de Salud. [Internet]. 1952 [citado 10 oct 2018]. Disponible en: https://tinyurl.com/ yyj3dw8p.

32. Urzúa H. Estructuración inicial del S.N.S. Revista Médica de Chile. 1977;105(10):659-660.

33. Bravo A. Servicio Nacional de Salud. Doctrina y política. Santiago: Servicio Nacional de Salud; 1962.

34. INSORA. Recursos humanos de la administración pública chilena. Santiago: INSORA; 1962.

35. Correa SS. Historia del siglo XX chileno. Santiago: Sudamericana; 2001.

36. Meller P. Un siglo de economía política chilena (1890-1990). Santiago: Editorial Andrés Bello; 1990.

37. Foxley A, Arellano JP. El tamaño y el papel del Estado. Santiago: CIEPLAN; 1977.

38. Muñoz O. El rol del Estado en el desarrollo de América Latina. Santiago: CIEPLAN, 1990.
39. Valenzuela A. El quiebre de la democracia en Chile. Santiago: FLACSO; 1978.

40. Lagos R. La concentración del poder económico. Santiago: Editorial del Pacífico; 1961.

41. Valenzuela A. Parties, politics, and the State in Chile: The higher civil service. En: Suleiman E, (ed.). Bureaucrats and policy making: A comparative overview. New York: Holmes\&Meier; 1984.

42. Goic A. El sistema de salud de Chile: una tarea pendiente. Revista Médica de Chile. 2015;143(6):778-779.

43. Medina E. Evolución de los indicadores de salud en el periodo 1960-1977. En: Lavados H, (ed.). Desarrollo social y salud en Chile. Tomo I. Santiago: CPU; 1979.

44. Medina E. Evolución de la salud pública en Chile en los últimos 25 años. Revista Médica de Chile. 1977;105(10):739-745.

45. Viel B. Principales índices biodemográficos y de salud en Chile entre 1950 y 1987. Santiago: APROFA; 1989.

46. Kaempffer AM. Evolución de la salud maternoinfantil en Chile, 1952-1977. Revista Médica de Chile. 1977;105(10):680-686.

47. Mardones Restat F. Quince años de labor del SNS. Revista Médica de Chile. 1967;95(8):475479.

48. McGuire J. Politics, policy, and mortality decline in Chile, 1960-1995. En: Salvatore R, Coatsworth JH, Challú AE, (eds.). Living standards in Latin America History: height, welfare and development. Cambridge: Harvard University-David Rockefeller Center for Latin American Studies; 2010.

49. Caldwell J. Routes to low mortality in poor countries. Population and Development Review. 1986;12(2):171-220

50. Raczynski D, Oyarzo C. ¿Por qué cae la tasa de mortalidad infantil en Chile? Santiago: CIEPLAN, 1981.

51. Colegio Médico de Chile, Departamento de Salud Pública. Servicio Nacional de Salud. Santiago: Colegio Médico de Chile; 1957.

52. Álvarez O. Trayectoria y proyecciones del Servicio Social en el Servicio Nacional de Salud. Santiago: Escuela de Salubridad Universidad de Chile; 1966. 
53. Ministerio de Salud Pública, Consejo Nacional Consultivo de Salud. Recursos Humanos de Salud en Chile. Santiago de Chile: Ministerio de Salud Pública; 1970.

54. Ducci H. Editorial. Revista Médica de Chile. 1959;87(5):321-323.

55. Servicio Nacional de Salud. Normas para el Servicio Social. Boletín del Servicio Nacional de Salud. 1956;II(3):358-369.

56. Wiegand G, Ramírez E. Evolución del hospital en Chile y servicio social de colaboración médica en un hospital general. Santiago: Universidad de Chile, Escuela de Servicio Social; 1954.

57. Lillo N, Roselló E. Manual para el Trabajo Social comunitario. Madrid: Narcea; 2004.

58. Lipsky M. Street-level bureaucrats: Dilemmas of the individual in public services. New York: Russel Sage Foundation; 1980.

59. Codoh Chávez E, Montecinos Peña R, Oportus Venegas A, Silva Hennings A. El Servicio Social en el medio rural. [Memoria de Prueba]. Concepción: Universidad de Chile, Facultad de Ciencias Jurídicas y Sociales, Escuela de Servicio Social; 1959.

60. Zárate MS. Señora, su hijo no va a morir: Enfermeras y madres contra la mortalidad infantil, Chile 1950-1980. En: Cordero-Fernández M, Moscoso-Flores PE, Viu A, (eds.). Rastros y gestos de las emociones. Santiago: Editorial Universidad Adolfo Ibáñez; 2018.

61. Graham M. Emotional bureaucracies: emotions, civil servants, and immigrants in the Swedish Welfare State. Ethos. 2002;30(3):199-226.

62. Honig M. Street-level bureaucracy revisited: frontline district central-office administrators as boundary spanners in education policy implementation. Educational Evaluation and Policy Analysis. 2006;28(4):357-383.

63. Kaplan I, Molina M. Servicio Social en el Centro de Salud de Viña del Mar y su aporte a la comunidad. [Memoria de Prueba]. Valparaíso: Universidad de Chile, Facultad de Ciencias Jurídicas y Sociales, Escuela de Servicio Social; 1954.

64. Servicio Nacional de Salud. Distribución de matronas. Boletín del Servicio Nacional de Salud. 1966;III(33):13-14.

65. Servicio Nacional de Salud. Distribución de matronas. Boletín del Servicio Nacional de Salud. 1966;III(34):2.
66. Servicio Nacional de Salud. Programa de Distribución de Matronas. Boletín del Servicio Nacional de Salud. 1966;/II(34):11-14.

67. Pérez ME. Sistematización de la práctica profesional realizada en el Servicio Nacional de salud, Consultorio Rosita O'Hlggins. Concepción: Universidad Católica de Chile, Escuela de Trabajo Social; 1975

68. Hidalgo O. La labor del servicio social profesional en colaboración con los programas de trabajo del Centro de Salud Independencia. Santiago: Universidad de Chile, Escuela de Servicio Social; 1956.

69. Alarcón M. Sistematización de práctica realizada en el Consultorio Materno-Infantil "San Vicente" de Talcahuano, del Servicio Nacional de Salud. Concepción: Universidad Católica de Chile, Escuela de Trabajo Social; 1975.

70. Godoy L, Zárate MS. Trabajo y compromiso. Matronas del Servicio Nacional de Salud. Chile, 1952-1973. Revista Ciencias de la Salud. 2015;13(3):411-430.

71. Ortiz M. Chillán y los Servicios Sociales Profesionales. Santiago: Universidad de Chile, Escuela de Servicio Social; 1964

72. Larrañaga O. Participación laboral de la mujer en Chile: 1958-2003. En: Valenzuela JS, Tironi Barrios E, Scully T. El eslabón perdido: Familia, modernización y bienestar en Chile. Santiago: Taurus; 2006.

73. Servicio Nacional de Salud. Normas para la organización del trabajo de enfermería en el Servicio Nacional de Salud. Boletín del Servicio Nacional de Salud. 1956;II(6):801-815.

74. Servicio Nacional de Salud. Enfermería. Boletín del Servicio Nacional de Salud. 1956;II(6):799800.

75. Díaz S, Pardo H, Pessoa S. Estudio de los roles en el equipo de enfermería. Cuadernos Médico Sociales. 1970; XI(4):20-32.

76. Chile, Ministerio de Salud Pública y Previsión Social. Ley 10.223 de 17 diciembre 1951, sobre Estatuto para los médicos, cirujanos, farmacéuticos o químico-farmacéuticos y dentistas [Internet]. 1951 [citado 10 oct 2018]. Disponible en: https://tinyurl.com/yxopxx5f.

77. Sepúlveda C. Roles profesionales y atención de la salud. Cuadernos Médico Sociales. 1970;XI (3):14-22 
78. Leiva L. Experiencia de un médico general de Zona en Puerto Octay. Cuadernos Médico Sociales. 1969; X(2):28-32.

79. Jungjohann A. Acciones de salud integral realizadas por el equipo de salud del Hospital de Lonquimay 1969 a 1971. Cuadernos Médico Sociales. 1972;XIII(4):37-40.
80. Morales J. Formación de médicos y paramédicos en Chile. Antecedentes, problemas y perspectivas. Santiago: CELADE, Universidad de Chile; 1964.

\section{FORMA DE CITAR}

González Moya M, Zárate Campos MS. Trabajo, miserias y recompensas: Asistentes sociales, enfermeras y matronas

en la construcción de la política sanitaria chilena a mediados del siglo XX. Salud Colectiva. 2019;15:e2214. doi: $10.18294 /$ sc.2019.2214.

Recibido: 1 de marzo de 2019 | Versión final: 1 de julio de 2019 | Aprobado: 16 de julio de 2019

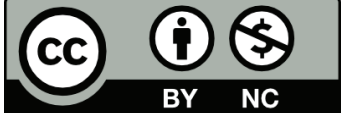

Esta obra está bajo una licencia de Creative Commons Reconocimiento-NoComercial 4.0

Internacional. Reconocimiento - Permite copiar, distribuir y comunicar públicamente la obra.

A cambio, se debe reconocer y citar al autor original. No Comercial - Esta obra no puede ser

utilizada con finalidades comerciales, a menos que se obtenga el permiso. 